\title{
Toward Fundamental Pollutant Routing Within Stormwater Control Measures Using Computational Fluid Dynamics
}

\author{
David W. Spelman ${ }^{1}$ and John J. Sansalone ${ }^{2}$ \\ ${ }^{1}$ Bradley University, Peoria, Illinois; ${ }^{2}$ University of Florida, Gainesville, Florida.
}

\begin{abstract}
Constituents such as sediment, nutrients and heavy metals carried by rainfall-runoff from urban surfaces pose an ecological threat to receiving water bodies and are thus increasingly regulated. Stormwater control measures such as wet basins, hydrodynamic separators and various green infrastructure unit operations are designed in part to separate constituents of concern from stormwater. The design, analysis and implementation of such systems, particularly innovative and unproven ones, requires a robust model capable of accurately predicting constituent load reduction given site-specific conditions. Many traditional empirical models assume that treatment systems behave as idealized continuously stirred or plug flow reactors and reduce constituent concentrations through first-order decay. While useful and historically necessary, given a lack of alternatives, this modeling approach has limited practical benefit in the design and implementation of innovative systems, and requires site-specific calibration to produce accurate results. Computational fluid dynamics has been used to simulate constituent transport and fate within stormwater unit operations in an effort to understand fundamental mechanisms, optimize design by improving volumetric utilization and providing performance predictions of design alternatives, and develop updated models for use in watershed planning. Recent modeling developments are presented together with a design example to demonstrate present challenges and future solutions.
\end{abstract}

\section{Introduction}

\subsection{Background}

Rainfall-runoff in urban areas produces substantial constituent loads, such as nutrients, metals and a wide size gradation of particulate matter, that are transported through drainage networks and into receiving water bodies. Suspended particulate matter (PM) is of concern since it is a vehicle for many constituents through partitioning and thus dictates transport and fate mechanisms for certain particle-bound pollutants such as lead and phosphorus. The potential negative impacts of stormwater on water quality and aquatic health are well documented (Lee and Bang 2000; NRC 2009). The treatment of stormwater and reduction of constituent loads is increasingly regulated. Stormwater control measures (SCM) represent critical infrastructure in managing stormwater quality and quantity by separating PM through gravitational sedimentation and providing residence time for biochemical decay processes to occur.

While used for decades, the underlying separation and decay mechanisms within systems such as wet detention basins and hydrodynamic separators remain ill understood due to the complex, unsteady and episodic nature of stormwater flows. Further complication is provided by the heterodisperse particle diameters that enter SCMs, as defined by the particle size distribution (PSD).
Additional complexity is provided by the significant geographic variability in loading conditions, environmental factors and water chemistry that result in corresponding variable treatment performance and response of SCMs (Koch et al. 2014). These combined effects make drawing conclusions about system design and expected performance challenging.

Regardless of the situation or mechanisms, two criteria for SCM design should be considered: the maximization of PM separation and constituent residence time. While system volume is the dominant factor in slowing down flow and provided residence time, volume alone is insufficient as a design parameter. Internal system hydrodynamics play an important role and describe the difference between constituent and theoretical residence time (Persson and Wittgren 2003). Systems must be designed to minimize short-circuiting and maximize volumetric utilization if they are to be optimally implemented (Garofalo et al. 2018). A primary hypothesis of this work is that traditional SCMs are suboptimal in volumetric utilization and thus do not provide the level of treatment that could be expected given their volume. The shape and inlet configuration of these systems, along with internal baffling and inlet diffusion represent design parameters that can be optimized to provide a desired treatment performance while minimizing volume and therefore cost. An illustration of this concept is shown in Figure 1. This optimization is only possible if a model is available to assess the effect of design alternatives. Such

Spelman, David W., and John J. Sansalone. 2018 Toward Fundamental Pollutant Routing Within Stormwater Control Measures Using Computational Fluid Dynamics. Journal of Water Management Modeling 26: C459. doi: 10.14796/JWMM.C459.

(c) CHI 2018. www.chijournal.org ISSN 2292-6062. 
a model must also be of minimal outlay such that the engineering cost of optimization does not exceed the expected benefit.

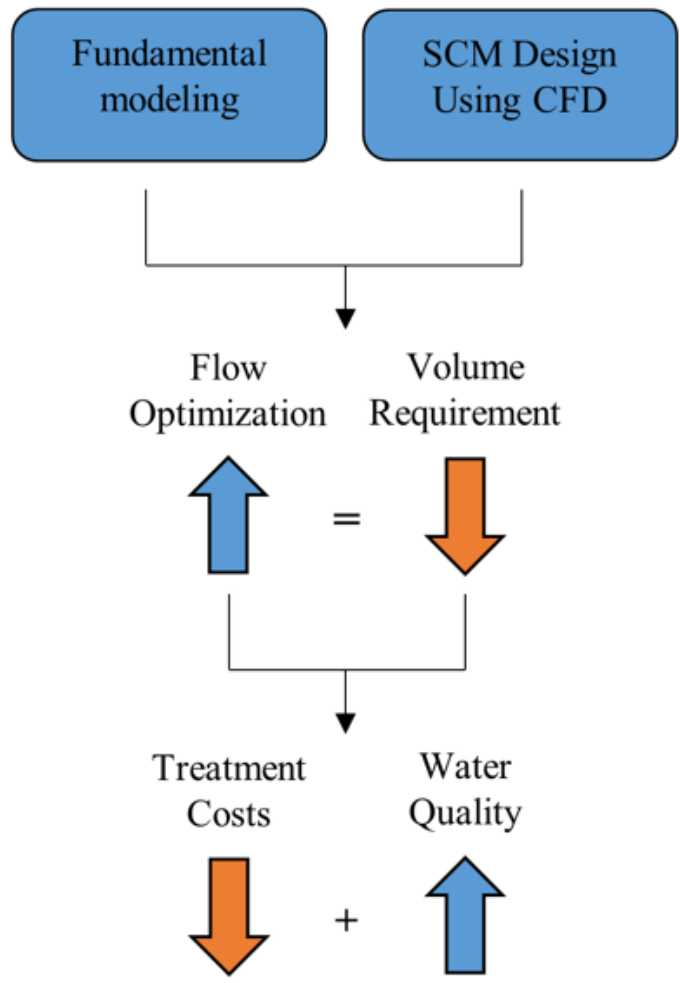

Figure 1 Justification and vision for CFD modeling of stormwater control measures.

\subsection{SCM Water Quality Modeling}

Traditional water quality routing models make necessary simplifying assumptions to approximate SCM load or concentration reduction using empirical equations. Approximations of SCMs as continuously-stirred tank reactors (CSTR) with treatment following first-order decay processes have been well utilized in stormwater modeling and can provide accurate results with calibration (Huber et al. 2006; Kadlec and Knight 1996). Such models are of limited use in design however as they provide no capacity for predicting the performance of design alternatives and innovative techniques and technologies. A fundamental physically-based simulation of constituent transport and fate within SCMs would allow for: (1) flow optimization and improved design of individual SCMs, potentially reducing cost and improving treatment efficiency; and (2) improved watershed planning in combining SCMs across a drainage network to meet regulatory goals such as total maximum daily load (TMDL). One tool that could offer a path forward is computational fluid dynamics (CFD) simulation.

In CFD simulation, SCMs are recreated in a 3D modeling application and meshed into a grid of individual cells; current modeling efforts use cell counts in the order of 10 million. The Reynolds averaged Navier-Stokes (RANS) fluid flow equations, with closure models, are numerically solved across this grid for a given flow condition, yielding a comprehensive representation of the flow field within the system. This process can be performed at steady flow rates or in a transient manner, stepping through time to simulate a storm event. The resulting flow field can be combined with Eulerian or Lagrangian models to track particle and dissolved constituent transport through the system. For particle tracking, a population balance approach can be used to assess the fraction of particles that are eluted from the system outlet versus trapped in sediment. Several, typically 8-16 (Dickenson and Sansalone 2009), individual particle diameters can be simulated to recreate the transport behaviour of the entire PSD.

Particles are represented as point objects with the diameter being used in calculation of drag, buoyancy and gravitational forces acting on the particles. Current methods do not model particleparticle interaction and thus only discrete settling is simulated. Some storm events may result in heavily particle-laden flows that produce flocculent or hindered settling which is not currently accounted for. The key results created from this modeling approach are PM separation efficiency, on a per-particle-size basis, and residence time distribution which quantifies the spectrum of constituent residence time that pollutants experience while traveling through the SCM. Decay models can also be incorporated to account for biochemical processes.

CFD is an extremely powerful tool, and has been shown to provide accurate prediction of PM load reduction for hydrodynamic separators when a transient simulation is performed for individual storm events coupled with extensive data input (Garofalo and Sansalone 2011). Such simulations require substantial computational resources (the equivalent of weeks to months of runtime on a workstation); robust input data, including flowrate, PM concentration and PSD, each as a function of time; and they are unique to each modeled event. A more practical alternative would need to allow for simulation of long term SCM behaviour with a decreased computational outlay relative to fully unsteady CFD.

\subsection{Objectives}

The objectives of this paper are fourfold. First, a vision for the implementation of CFD in stormwater treatment design and application, with potential benefits to the industry, is elucidated. Second, a brief overview of recent efforts in developing CFD modeling capability for stormwater SCMs is provided. Third, a design example is presented of a wet detention basin to show how fundamental simulation can allow for more effective stormwater treatment, thereby improving water quality and reducing runoff management costs. Finally, future research needed in the application of CFD to the stormwater field is discussed.

\section{Recent Model Developments}

An alternative to unsteady CFD simulation is offered by the stepwise-steady methodology, which relies on steady flow CFD results to reproduce unsteady transport by assuming that transient processes may be approximated by a series of steady flowrates. 
Use of the method to route PM through an SCM for a given input condition involves organizing input data of hydrograph, PSD, and PM concentration; obtaining data for the SCM of residence time distribution and PM removal as a function of flowrate either from CFD or traditional methods; and running a script. Widespread adoption of the method requires future effort in distributing the underlying code, templates, and documentation or building it into an existing software package.

Originally introduced by Pathapati and Sansalone (2011), the stepwise-steady method has been updated and applied to a variety of SCMs in recent years (Cho and Sansalone 2013; Spelman and Sansalone 2017; 2018). One development by Spelman and Sansalone (2018) demonstrated that steady flow treatment response results of manufactured clarifiers and hydrodynamic separators, modeled using CFD, are portable across geographic locations. By performing a series of steady flow CFD simulations, a normalized set of SCM performance metrics can be created that represents the response of the system to the complete set of expected conditions under steady flow. Spelman and Sansalone (2018) modeled two SCMs in CFD for various temperatures, flow rates, PM densities, and PM diameters to produce a normalized dataset of PM separation results for each system. These results were then combined with a stepwise-steady model to simulate the effluent PM load from three different design storms. Depending on the input conditions, PM separation efficiency of the systems ranged from $25 \%$ to $95 \%$. It was found that influent PSD has the largest impact on SCM load reduction. Portability was demonstrated through the prediction of load reduction for multiple input conditions using one set of underlying SCM performance results (Spelman and Sansalone 2018).

The robustness of CFD and stepwise-steady models was enhanced by Spelman and Sansalone (2017) with the development of an updated stepwise-steady methodology (the implicit solution stepwise-steady, or $\mathrm{IS}^{3}$, method) that increased the accuracy of recreating unsteady transport with only steady flow CFD results. The improvement arose from an addition that implicitly determined the flowrate that best represents unsteady transport at each time step given the set of flows a constituent would experience during transport through the system. The stepwisesteady methodology divided each simulated event into 2000 time increments. Fewer time steps may be used while maintaining accuracy. However, the short run time ( $<1 \mathrm{~min}$ ) of stepwisesteady models, implemented thus far as MATLAB scripts, did not warrant an optimization study (Spelman and Sansalone 2017). In addition, this work expanded the applicability of the method to large-volume treatment systems such as wet detention basins. The work also showed that single event load reduction analyses may provide non-representative results, particularly for large systems and small storm events. Spelman and Sansalone (2017) recommended a long-term simulation approach to designing and analysing SCMs.

\section{Design Example: Wet Basin Retrofit}

A wet detention basin located in the grounds of a Florida airport requires retrofit due to the removal of upstream treatment train capacity for runway expansion. Due to land availability and cost restrictions, an innovative approach to add baffles to the existing pond is suggested. A baffled system may allow for increased constituent residence time by reducing short-circuiting and improving volumetric utilization. No model exists, however, to confidently predict the effect of baffle addition on constituent load reduction, or to determine the optimal number of baffles to add in balancing performance and cost. Complexity is increased by the need to maintain a $0.5 \mathrm{~m}$ freeboard over the baffle surface, to limit bird attraction, for aircraft safety.

A CFD model of the existing basin is constructed together with models of the basin with several configurations of internal baffling designed to improve volumetric utilization and increase constituent residence time. An isometric image of the baffled system in shown in Figure 2 along with detailed properties. The baffles are $1 \mathrm{~m}$ thick porous gabion walls that allow the passage of some flow, modeled in CFD using the Ergun equation. This porous system may also provide additional treatment by providing a surface for attached growth; however, future research is needed to quantify this effect.

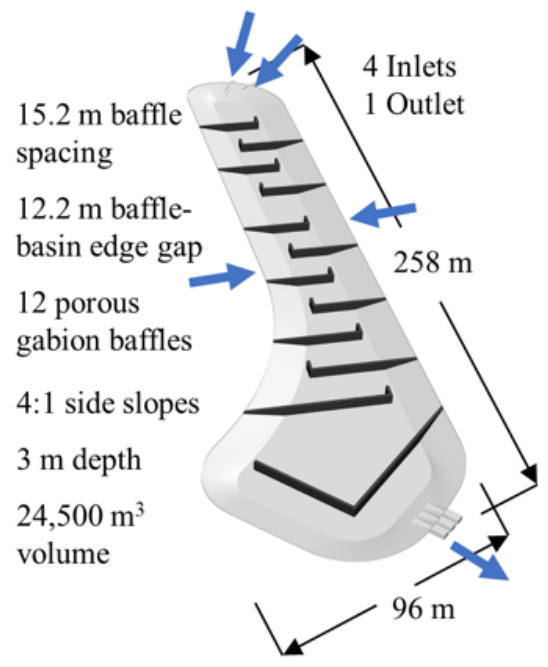

Figure 2 Dimensioned and isometric image of the example basin with retrofit.

A series of steady flow CFD simulations are performed to obtain a set of performance results giving constituent residence time distribution and particulate matter removal as a function of flowrate. The number of steady flowrates simulated must be sufficient to well characterize the trends in results with respect to flowrate; in this example, 11 flowrates were modeled for each design. This set of CFD results provides a signature of the modeled system in terms of its treatment response to the range of flowrates it is expected to experience. This set of results is produced for each design alternative. A comparison of steady flow rate fluid velocity contours between the existing and retrofitted basin is 
shown in Figure 3. A similar comparison is made in Figure 4 to show the particle trajectories of a tracer (neutrally buoyant) particle colored by residence time. Only one baffled design is shown in this paper, however many alternatives can be developed and simulated for optimization.

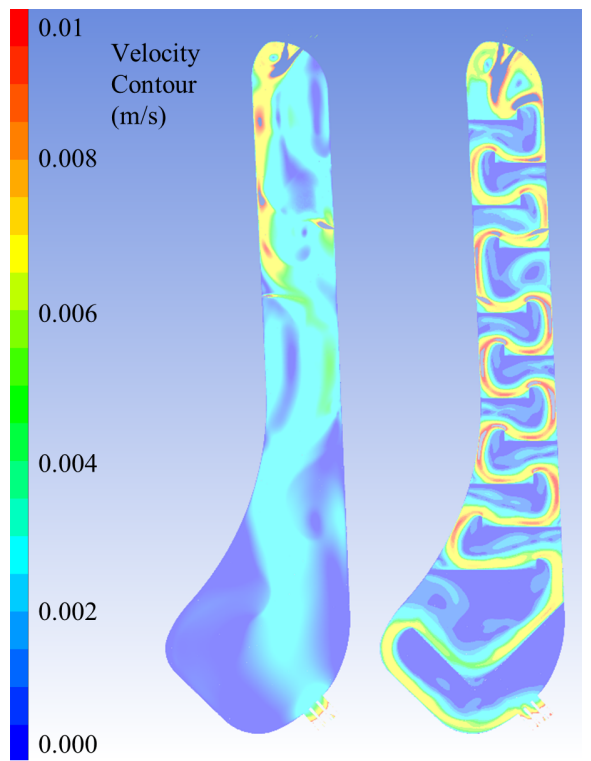

Figure 3 Velocity contours at mid-depth in the example basin before and after baffle retrofit (shown for a $100 \mathrm{~L} / \mathrm{s}$ flow rate).

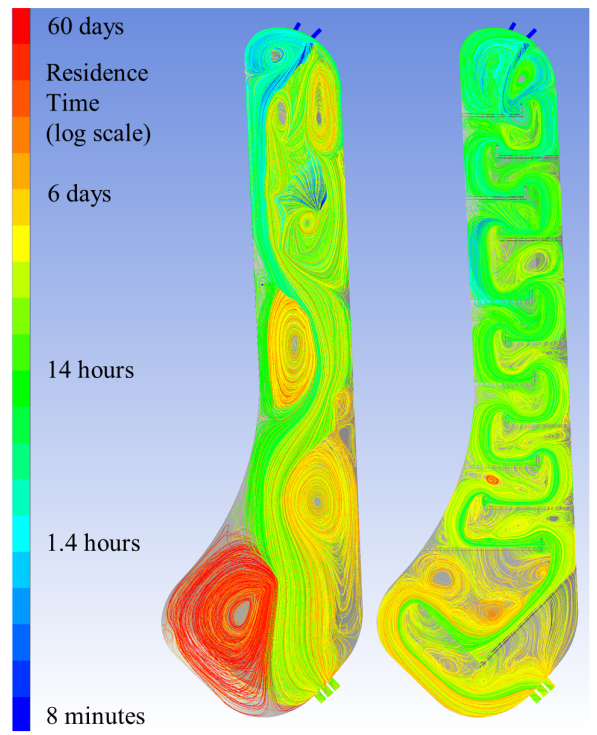

Figure 4 Tracer particle trajectories colored by residence time in the example basin before and after baffle retrofit (shown for a $100 \mathrm{~L} / \mathrm{s}$ flow rate; median residence time without retrofit is $31 \mathrm{~h}$, and with retrofit is $58 \mathrm{~h}$ ).

The CFD results are combined with a stepwise-steady model to create a long term simulation of performance using historical rainfall data and a hydrologic model as additional inputs.
The long term (for example $1 \mathrm{y}$ ) load reductions of each design alternative can then be compared and evaluated against their relative costs. The effect of the selected retrofit can also be quantified, using modeled results, against the existing basin, to justify the cost of the retrofit and provide reasonable assurance that sufficient improvement to the stormwater treatment system has been made to achieve water quality goals. Current models remain relatively new and unproven, and thus a monitoring campaign is additionally needed to validate modeled results and ensure that sufficient treatment is occurring within innovative systems such as baffled wet basins. Current modeling limitations, as outlined in the following section, do not allow for the accurate and robust predication of effluent loads as described above for such large systems. Extensive site-specific monitoring with calibration and verification are still needed for accurate results on an absolute basis. Rather, relative differences in performance indicators, such as constituent residence time, between design alternatives are the useful result of CFD modeling efforts given current methods and understanding.

In this example, the ANSYS Fluent software package was used for CFD simulation, with ANSYS Meshing used for computational grid generation. The mesh for each geometry consisted of 9-11 million computational cells. A grid independence study, excluded here for brevity, was performed to determine the mesh number necessary to reduce grid-related error below a tolerance of $1 \%$ as measured by median particle residence time. The realizable $k$-epsilon turbulence model with enhanced wall functions was used alongside the Reynolds averaged Navier-Stokes (RANS) equations. The water free surface was fixed and specified as a zero-shear boundary condition. Approximately 2000 tracer particles were injected and tracked through the system, as shown in Figure 4. The methods used resulted in computational run times of approximately $2 \mathrm{~d}$ (16 CPU-days) per steady flow simulation using 8 Intel E5-2698 v3 processors.

Simulating two designs, with 11 steady flows each, required 352 CPU-days of computational effort. Any long-term simulation scenario can then be performed using stepwisesteady with negligible computational effort (<0.1 CPU-days). The computation effort associated with producing a full set of steady flow results is on the same order as that required for an unsteady (transient) CFD simulation of a single storm event (Spelman and Sansalone 2017). Long term simulation using unsteady CFD is therefore impractical, and the benefit of the stepwise-steady methodology is thus demonstrated.

\section{Future Direction}

To realize the potential of CFD in the field of stormwater treatment, significant hurdles need to be overcome through future research. In this field CFD is a relatively recent development tool and has a long way to go before being widely adopted. In this section, a few key research needs will be identified and discussed concerning obstacles to the realization of fundamental pollutant routing through SCMs. 


\subsection{Data Input}

Models are limited in accuracy by the breadth and quality of the input data they are given. Certain input data are more readily available than others. Input hydrographs for example are obtained with relative ease for a given catchment using modern hydrologic modeling software. Other input parameters that affect the treatment response of SCMs, such as water temperature (Spelman and Sansalone 2018) may reasonably be estimated.

Quantification of influent suspended sediment concentration, or the concentration of other constituents of concern, as a function of time throughout the hydrograph is necessary for data input. Washoff models, potentially those that include buildup, are integrated into hydrologic modeling software packages and may provide accurate results with site-specific calibration (Obropta and Kardos 2007; Wijesiri et al. 2016). Inaccuracies in input concentration data will affect the predicted influent, and therefore effluent load; however, the predicted treatment response, as a percentage reduction in load, may be relatively unaffected compared with other data input uncertainties. This is true for PM separation because sedimentation occurring in stormwater unit operation is largely discrete settling in which settling velocity is independent of concentration (Dickenson and Sansalone 2009). This may not hold for all cases, and the treatment response of dissolved species which may undergo biochemical reactions that are dependent on concentration will be affected by inaccuracies in input concentration data. Additionally, the time distribution of influent PM concentration within a storm (presence or absence of a first flush for example) will affect the percentage load reduction due to differences in flowrates experienced by majority of influent PM.

For stormwater treatment, it has been demonstrated that influent PSD is the most critical parameter in determining PM load reduction in SCMs (Spelman and Sansalone 2018). To our knowledge, there is currently no predictive model to estimate PSD in runoff. There is a need for the development of a predictor of stormwater PSD that is based on geographic location, site conditions such as land use and soil characteristics, and other measurable environmental factors. PSD may change as runoff is transported overland and through a distribution network because the coarsest particles may not be transported. This represents an additional challenge when providing input PSD data to a SCM placed within a storm sewer network. Further model development is needed to predict change in PSD as stormwater flows downstream. PSD has been shown to be the most important factor affecting SCM performance, and a practical tool is needed to provide a reasonable estimate of PSD given realistically attainable measurements for a given site.

To fully understand fundamental pollutant routing, as illustrated in the concept routing image in Figure 5, the partitioning of metals and nutrients into and away from PM must be well understood. Partitioning does not occur uniformly across the PSD, and a disproportionate mass of constituents is typically associated with the finer fraction of PM (Sansalone and Tribouillard
1999; Djukić et al. 2016; Lau and Stenstrom 2005). The nature of this partitioning is a complex function of PM surface morphology, mineralogy and PSD as well as the nature of the specific metal or nutrient of interest and its concentration, and water chemistry parameters such as $\mathrm{pH}$, oxidation reduction potential, pavement residence time and other constituent concentrations (Huber et al. 2016; Sansalone and Buchberger 1997). There is a need for improved understanding and better modeling methodologies for metal and nutrient partitioning during washoff, transport through drainage networks and treatment systems, and within SCMs during quiescent conditions. A model developed as envisioned by Figure 5 could take the form of a set of submodels built into a CFD simulation, or as a lumped companion model that utilizes CFD output information such as residence time distribution. Many strategies could be developed depending on the breadth of data available, and the desired level of complexity to be captured. Further research is needed to determine the feasibility and accuracy of various approaches.

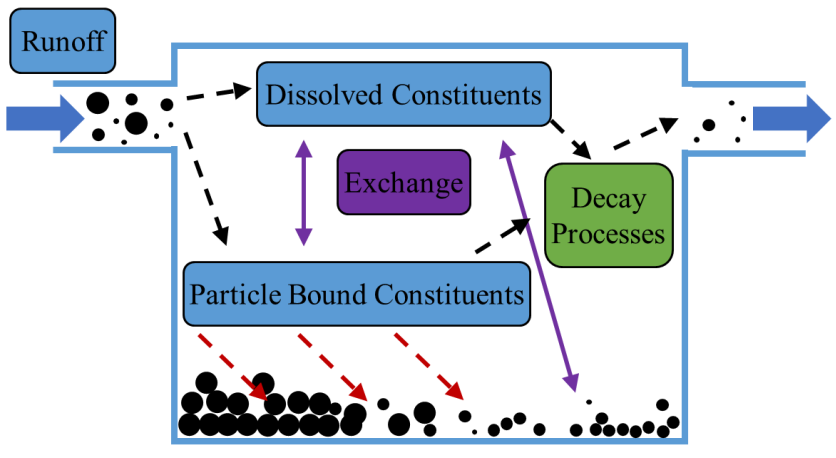

Figure 5 Conceptual routing diagram for constituents traveling through stormwater control measures by categorization into dissolved or particle-bound phases which undergo different processes; exchange between the two phases, both from suspended and settled sediment, is expected along with biochemical decay processes but the exact nature of these interactions is dependent on a variety of site- and storm-specific conditions.

\subsection{CFD Simulation}

Further development and understanding of CFD models, as they are applied to stormwater treatment, is necessary for widespread use of the tool. Many current implementations, both steady and unsteady, assume that SCMs maintain a fixed volume throughout a storm event. Future research toward the development of volume of fluid (VOF) CFD simulations, or similar approaches, which allow the free water surface to vary continuously would allow for further application. Such efforts could also quantify the error associated with constant volume assumptions with respect to treatment response, load reduction and residence time distribution. 
Further development of CFD methodology as applied to large scale systems such as wet basins used to treat stormwater would also be beneficial. In large systems, having a large surface area relative to volume, wind induced turbulence may have a substantial effect on internal basin hydrodynamics, constituent transport processing, and particulate matter settling. These effects may represent a significant source of error in current generation models that do not account for such processes. It is expected that wind induced turbulence would have the effect of retaining PM in suspension relative to a quiescent condition. If proven true, this would indicate that models that do not account for wind induced turbulence overpredict PM separation. Furthermore, developed CFD simulations of basins using a VOF approach may be able to directly simulate wind induced turbulence, and its effects on PM transport, by supplying a source term shear force at the free surface boundary. Future research is needed in this area. Cyclical changes in temperature may additionally impact PM settling as well as constituent partitioning and reactions, which may need to be considered in systems with long residence times.

\subsection{Companion Models}

To be useful in the design and implementation of SCMs, CFD simulation cannot be used in a vacuum; rather, CFD results must be used in conjunction with companion models. One such combination is the use of a stepwise-steady model to reproduce unsteady constituent transport and fate using steady flow CFD results. As previously discussed, this methodology has been demonstrated to provide accurate results for small manufactured SCMs (Cho and Sansalone 2013) and can be applied to larger systems with appropriate modification (Spelman and Sansalone 2017). Further development of the stepwise-steady methodology is needed to increase understanding of the unsteady constituent transport process and correspondingly improve modeling strategy. In addition, validation of the stepwise-steady methodology to long term simulation of designed treatment systems, including interevent processes is needed. With further development, stepwise-steady methods may be a useful tool for predicting long term SCM performance in a computationally efficient manner. A final stage in model development could be the incorporation of stepwise-steady methodology into popular hydrologic models, such as the USEPA's Stormwater Management Model (SWMM). This would involve integrating stepwise-steady into the water quality sub-model to route pollutants through storage systems. Stepwise-steady methods, combined with the results from steady flow CFD simulations, could be utilized by end users.

A hurdle to widespread use of CFD in stormwater treatment is in the specialized nature of performing CFD simulation and the associated engineering and computational outlay. An area of future research could be a meta-analysis from detailed study and CFD simulation of many traditional SCMs, as well as innovative designs. Such an effort might result in updated best practices and guidelines for optimizing volumetric utilization. This practical guideline could be used in new construction and retrofit applications without the need for site-specific CFD simulation, while potentially gaining some or most of the benefits.

One potential roadmap for future progress toward the goal of fundamental pollutant routing through SCMs may be summarized as follows:

1. Perform research into the basic science of contaminant transport both in runoff and within SCMs.

2. Develop sophisticated models capable of simulating these processes.

3. Develop simplified models that approximate the current expensive (in terms of computation and data input) simulations, with clear bounds on accuracy and applicability.

4. Integrate simplified models into commonly used hydrologic modeling software such as SWMM.

5. Apply the preceding to new systems and SCM retrofit to improve load reduction performance and reduce the cost of stormwater treatment.

\section{Conclusions}

Computational fluid dynamics is a tool with great potential to aid the field of stormwater treatment. Inefficiencies in treatment systems design that are due to a lack of volumetric utilization caused by shape and inlet configurations may lead to non-optimal spending on stormwater infrastructure or worse than expected treatment performance. A fundamental predictive tool such as CFD could offer a path towards the design and implementation of innovative control measures that have optimized internal hydrodynamics to maximize treatment efficiency at minimal outlay. Fundamental models may also provide more robust and accurate predictions of effluent loads than are currently available. While recent developments have made progress toward the implementation of CFD in the field of stormwater treatment, a significant investment in future research is needed to realize its potential.

\section{References}

Cho, H. C., and J. J. Sansalone. 2013. "Instantaneous Stepwise-Steady CFD Model of BMP Response to Unsteady PM Loadings." Journal of Environmental Engineering 139 (11): 1350-60.

https://doi.org/10.1061/(ASCE)EE.1943-7870.0000749

Dickenson, J. A., and J. J. Sansalone. 2009. “Discrete Phase Model Representation of Particulate Matter (PM) for Simulating PM Separation by Hydrodynamic Unit Operations." Environmental Science and Technology 43 (21): 8220-6.

Djukić, A., B. Lekić, V. Rajaković-Ognjanović, D. Veljović, T. Vulić, M. Djolić, Z. Naunovic, J. Despotović and D. Prodanović. 2016. “Further Insight into the Mechanism of Heavy Metals Partitioning in Stormwater Runoff." Journal of Environmental Management 168:104-10. https://doi.org/10.1016/j.jenvman.2015.11.035

Garofalo, G., and J. J. Sansalone. 2011. “Transient Elution of Par- 
ticulate Matter from Hydrodynamic Unit Operations as a Function of Computational Parameters and Runoff Hydrograph Unsteadiness." Chemical Engineering Journal 175 (1): 150-9.

https://doi.org/10.1016/j.cej.2011.09.086

Garofalo, G., J. J. Sansalone and M. Asce. 2018. “Urban Drainage Clarifier Load-Response as a Function of Flow, Unsteadiness, and Baffling." Journal of Environmental Engineering 144 (3): $1-9$. https://doi.org/10.1061/(ASCE)EE.1943-7870.0001283

Huber, M., A. Welker and B. Helmreich. 2016. “Critical Review of Heavy Metal Pollution of Traffic Area Runoff: Occurrence, Influencing Factors, and Partitioning." Science of The Total Environment 561:895-919. https://doi.org/10.1016/j.scitotenv.2016.04.051

Huber, W. C., L. Cannon and M. Stouder. 2006. BMP Modeling Concepts and Simulation. U.S. Environmental Protection Agency Office of Research and Development.

Kadlec, R. H., and R. L. Knight. 1996. Treatment Wetlands. Boca Roton, FL: CRC Press.

Koch, B. J., C. M. Febria, M. Gevrey, L. A. Wainger and M. A. Palmer. 2014. "Nitrogen Removal by Stormwater Management Structures: A Data Synthesis." Journal of The American Water Resources Association 50 (6). https://doi.org/10.1111/jawr.12223

Lau, S. L., and M. K. Stenstrom. 2005. "Metals and PAHs Adsorbed to Street Particles." Water Research 39 (17): 4083-92. https://doi.org/10.1016/j.watres.2005.08.002

Lee, J. H., and K. W. Bang. 2000. “Characterization of Urban Stormwater." Water Research 34 (6): 1773-80.

NRC (National Research Council ). 2009. Urban Stormwater Management in the United States. Washington, DC: National Academies Press. https://doi.org/10.17226/12465
Obropta, C. C., and J. S. Kardos. 2007. "Review of Urban Stormwater Quality Models: Deterministic, Stochastic, and Hybrid Approaches." Journal of the American Water Resources Association 43:1508-23.

Pathapati, S. S., and J. J. Sansalone. 2011. “Can a Stepwise Steady Flow Computational Fluid Dynamics Model Reproduce Unsteady Particulate Matter Separation for Common Unit Operations?" Environmental Science and Technology 45 (13): 5605-13.

Persson, J., and H. B. Wittgren. 2003. "How Hydrological and Hydraulic Conditions Affect Performance of Ponds." Ecological Engineering 21 (4-5): 259-69. https://doi.org/10.1016/j.ecoleng.2003.12.004

Sansalone, J. J., and S. G. Buchberger. 1997. "Partitioning and First Flush of Metals in Urban Roadway Storm Water." Journal of Environmental Engineering 123 (2): 134-43. https://doi.org/10.1061/(ASCE)0733-9372(1997)123:2(134)

Sansalone, J. J., and T. Tribouillard. 1999. "Variation in Characteristics of Abraded Roadway Particles as a Function of Particle Size." Transportation Research Record 1690:153-63.

Spelman, D., and J. J. Sansalone. 2017. "Methods to Model Particulate Matter Clarification of Unit Operations Subject to Unsteady Loadings." Water Research 115:347-59. https://doi.org/10.1016/j.watres.2017.02.053

Spelman, D., and J. J. Sansalone. 2018. "Is the Treatment Response of Manufactured BMPs to Urban Drainage PM Loads Portable?" Journal of Environmental Engineering 144 (4). https://doi.org/10.1061/(ASCE)EE.1943-7870.0001326

Wijesiri, B., P. Egodawatta, J. Mcgree and A. Goonetilleke. 2016. "Understanding the Uncertainty Associated with Particle-Bound Pollutant Build-up and Wash-off: A Critical Review." Water Research 101:582-96. https://doi.org/10.1016/j.watres.2016.06.013 